\title{
Analysis of Ground Vibration near a Railroad
}

\author{
Katou Masafumi* Kanda Hitoshi** Sanada Yoshinori* Matsuoka Toshifumi* \\ *Kyoto university $\quad{ }^{* *}$ Central Japan Railway Company
}

\begin{abstract}
The ground vibration generated by the Shinkansen, Japanese super express train, can be problematic for people and structures nearby. The purpose of this study is to make clear the mechanism of the ground vibration generated by a train and to develop a numerical simulation tool which can quantitatively evaluate the effect of wave filtering. This study is consist with 2 parts: First, the Doppler effect in the field vibration data from train motion is shown to be found via FK spectrum. Second, the generation of ground vibration is classified by this numerical simulation. The ground vibration is caused by inhomogeneous media, inhomogeneous force from the train or discrete structure, like an elevated bridge. This work does not investigate the boundary problem between the train body and the ground, thus is a qualitative basic study.
\end{abstract}

Key Words : ground vibration, train, Doppler effect, numerical simulation

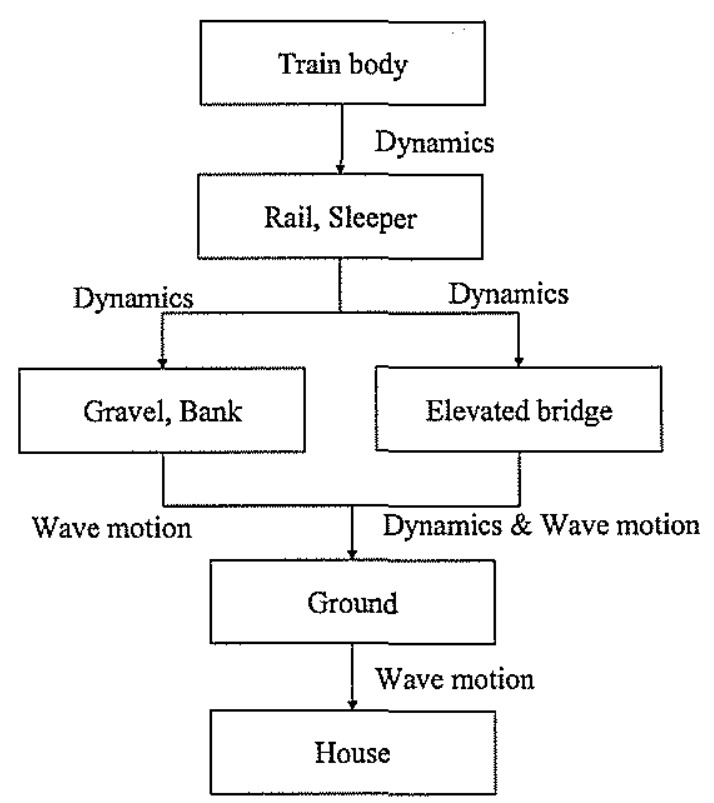

Figure 1 Wave route.

\section{Introduction}

Trains generate ground vibration as in Fig1. The mechanism can be divided 3 stages ; 1,Source(characteristics), 2,Path(property of media) and 3,Structure(dynamics of structures). For example we can apply wave filtering in each stage ; 1, Lightening the train body for source, 2,Excavating a gutter for path, 3 ,Using viscous material for structure. Every wave filtering method is uncertain. Because the ground vibration generated by a train is not investigated clearly. However it is very diff-

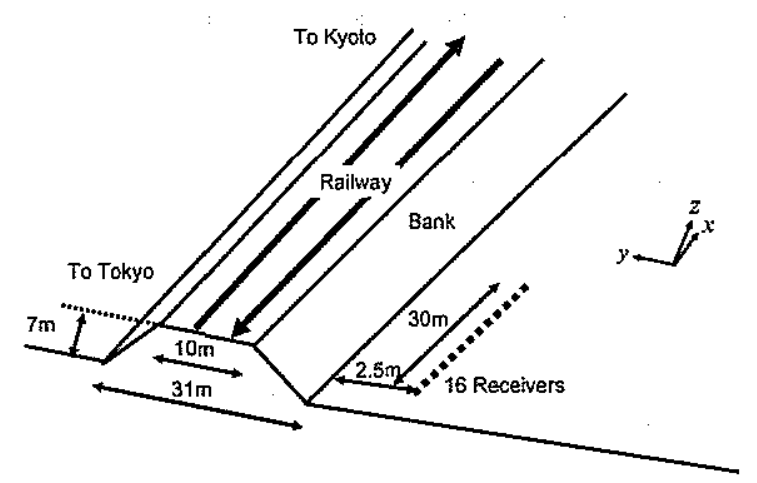

Figure 2: Schematics of the railway lines on bank and arrangement of receivers.

cult to solve boundary problems(Dynamics in Fig1) like between train body and the ground, propagation of vibration is obviously based on wave equation. Recently technologies of electronics developed rapidly. Today we can calculate Green function easily and Parallelization is not difficult. It is effective to classify the vibration types before studying the boundary problems.

Yoshioka and Ashiya(1995) ${ }^{1)}$ shows the vibration is not caused static but dynamic for direction. We analyze field data and propose some causes by numerical simulation.

\section{Analysis from field data}

Sinkansen has 16 cars and 64 shafts, In this paper we investigate the response from one wheel. Because in case of simulation, we can make the response from all wheels by superposition from one wheel. Thus it 


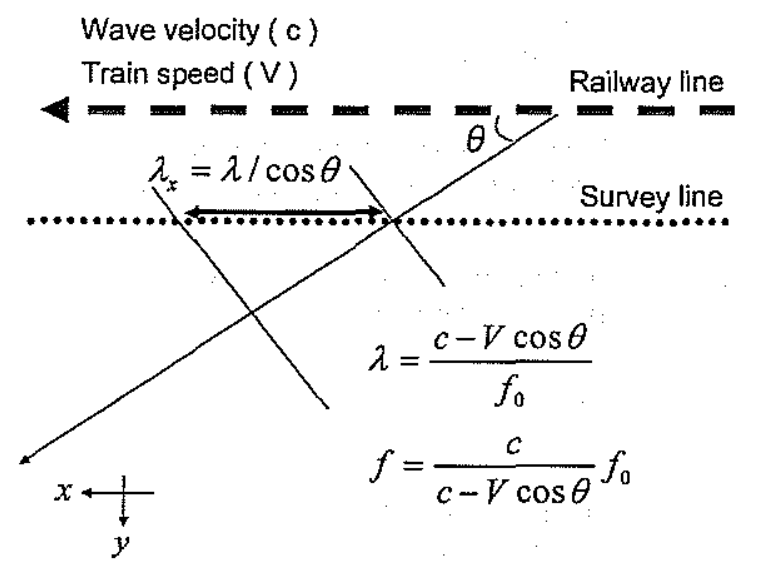

Figure 3: Schematics of direction of waves and equation of Doppler effect.

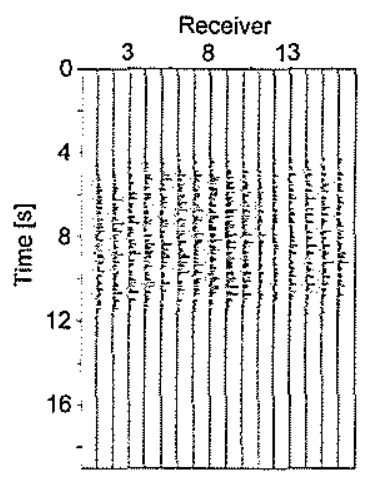

Figure 4: Waveforms from the train Tokyo to Kyoto.

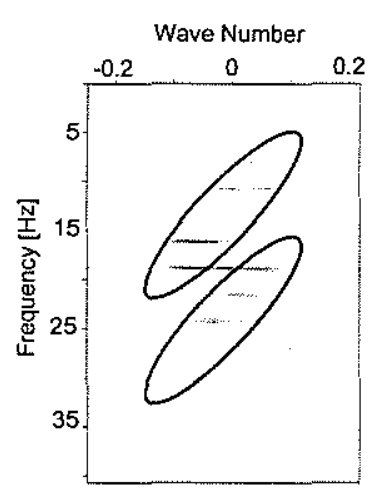

Figure 5: FK spectrum of Fig4.

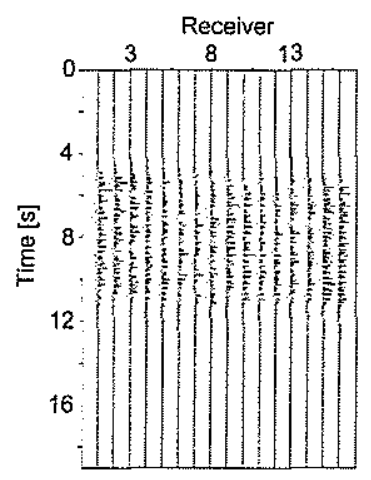

Figure 6: Waveforms from the train Kyoto to Tokyo.

Figure 7: FK spectrum of Fig6. is important to realize the vibration from one wheel when we analyze the field data.

Fig2 shows the shape of bank and arrangement of receivers. If we investigate Doppler effect in the field data, the source of the vibration is not from point but distributed. In case of $1 \mathrm{D}$ arrangement like Fig3, Doppler effect shows the following easy equation via FK spectrum.

$$
f=V k_{x}+f_{0}
$$

Where, $\mathrm{f}$ is frequency observed. $\mathrm{V}$ is train speed. $k_{x}$ is wave number in survey line. $f_{0}$ is frequency at stationary condition.

Fig4 shows the waveform from train Tokyo to $\mathrm{Ky}-$ oto. Fig5 shows FK spectrum of Fig4. Fig6 shows the waveform from train Tokyo to Kyoto. Fig7 shows FK spectrum of Fig6. Each slope of FK spectrum ( ellipses in Fig5 or Fig7) matches each train speed. Thus the field data observe Doppler effect.

We investigated that Doppler effect must be observed in numerical simulation. But the mechanism of the vibration is not realized perfectly. We should classify variable sources and models in numerical simulation.

\section{Numerical simulation}

\section{Methods}

We use the viscoelastic wave equation ${ }^{2}$ ) in order to hanlde the attenuation of vibration. And numerical calculation method is the explicit finite difference time domain method. To handle huge scale 3D modeling, we apply parallelization using PC-cluster. We use 8 nodes PC-cluster. $320 \times 160 \times 80$ grids, and 8000 steps scale can be calculated for 4 hours. It is 9 times faster than using one PC. It improved our efficiency of many numerical case studies.

\section{Classification of source}

Qualitative properties (Doppler effect ) of ground vibration are observed from analysis of field data. Following 6 cases are tested varying force from train body, media propagating wave and structure touching ground discretely.

1. Synchronized force (Fig8) and homogeneous media ( Fig10)

2. Synchronized force (Fig8) and inhomogeneous media ( Fig11)

3. Synchronized force (Fig8) and elevated bridge ( Fig13)
4. Not synchronized force (Fig9) and homogeneous media ( Fig10) 

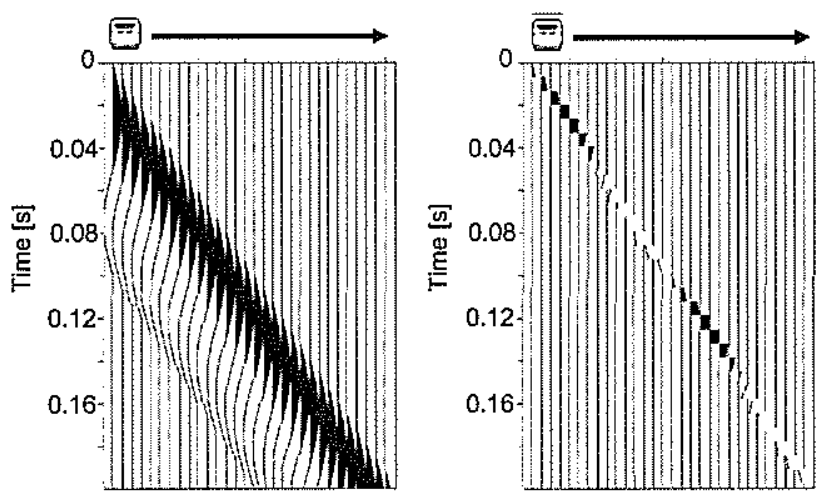

Figure 8: Waveforms of synchronized input.
Figure 9: Waveforms of Not synchronized input.

5. Not synchronized force ( Fig9) and inhomogeneous media ( Fig11)

6. Not synchronized force ( Fig9) and elevated bridge ( Fig13)

Secondary wave velocity ( Vs ) of the ground of homogeneous media is $150 \mathrm{~m} / \mathrm{s}$.

Considering Kamei, et al (2004) ${ }^{3)}$, we make inhomogeneous media which has $5 \mathrm{~m}$ correlation distance for 3D distance component. Fig11 is ; Step1 : Make 2D $5 \mathrm{~m}$ correlation distance in horizontal plane, $\mathrm{Vs}=170 \sim 230 \mathrm{~m} / \mathrm{s}$. Step2 : Make horizontal plane each $5 \mathrm{~m}$ in vertical direction. Step3: Cubic interpolation in vertical direction. However, receiver is too near for vibration to propagating inhomogeneous media enough ( Circle in Fig12 shows this condition. ). Therefore both the field data and simulation data are scarcely affected by inhomogeneous media in terms of propagation route. It is more important to analyze the relationship between the response at receiver and inhomogeneity near the railroad.

Synchronized force means same phase of waveform, therefore we define input waveform Fig8. Not synchronized force means train body is vibrating at $10 \mathrm{~Hz}$, therefore we define input waveform Fig9.

Elevated bridge also should be considered, since elevated bridge is the structure touching the ground discretely.

\section{Results}

We investigate followings by numerical simulation.

1. Synchronized force and homogeneous media can not generate ground vibration because of wave superposition ( Fig14, Fig15).

2. Synchronized force and inhomogeneous media generates ground vibration ( Fig16, Fig17 ). The frequency of ground vibration shows Doppler effect following $\mathrm{Eq}_{\mathrm{q}}(1)$. This model has 2 key points. First, the wave pathes are not affected by inhomogeneity in Fig12. Secondly, the ground vibration is mainly generated by inhomogeneity near the track. Fig26 shows that peeks in spectrum at wave number 0 in Fig17 matches (Vs parameter under the railroad) / (Train speed).

3. Synchronized force and elevated bridge generate the ground vibration of eigen-frequency ( Fig18, Fig19). The train speed is $75 \mathrm{~m} / \mathrm{s}$. Leg interval of the bridge is $6 \mathrm{~m}$. Thus the eigen-frequency of this case is $8.3 \mathrm{~Hz}, 12.5 \mathrm{~Hz}, 25 \mathrm{~Hz}$ and so on.

4. Not synchronized force and homogeneous media generate the ground vibration because of train's vibration ( Fig20, Fig21).

5. Not synchronized force and inhomogeneous media generate the ground vibration because of train's vibration ( Fig22, Fig23 ). Inhomogeneity of media scarcely affects the ground vibration.

6. Not synchronized force and elevated bridge generate the ground vibration of eigen-frequency ( Fig24, Fig25).

In case 2 to case 6 could observe ground vibration. 


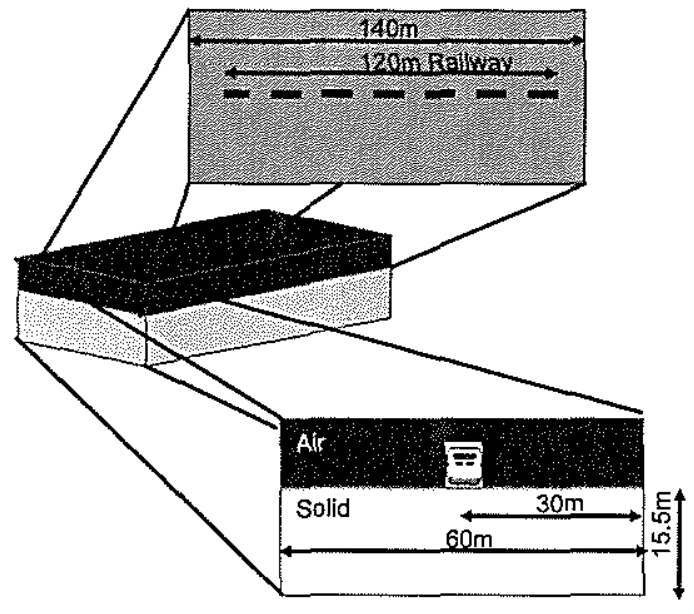

Figure 10: Schematics homogeneous media model.
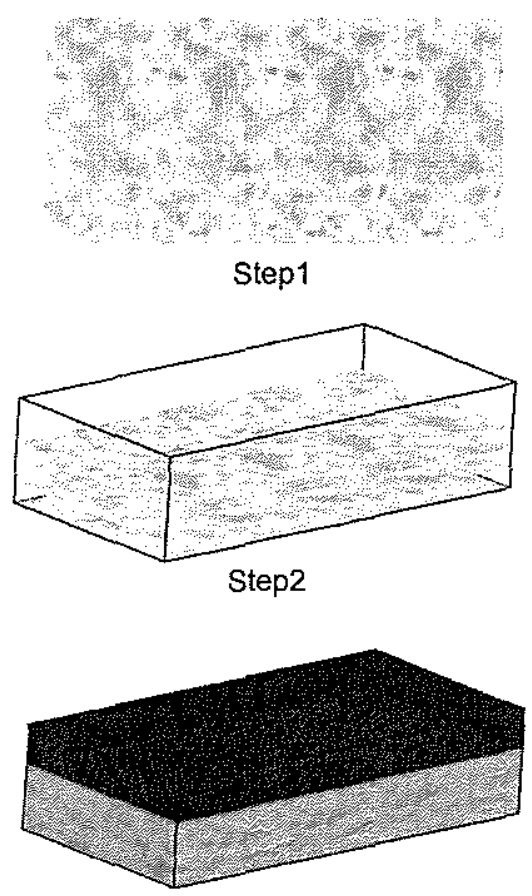

Step3

Figure 11 Inhomogeneous media model.

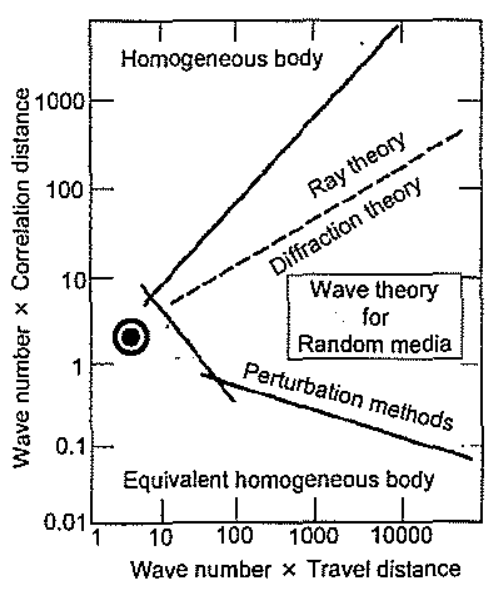

(Aki \& Richards, 1980)

Figure 12: Wave classification by Wave number, Correlation distance and Travel distance in inhomogeneous media(Aki and Richards, 1980) ${ }^{4}$ ).
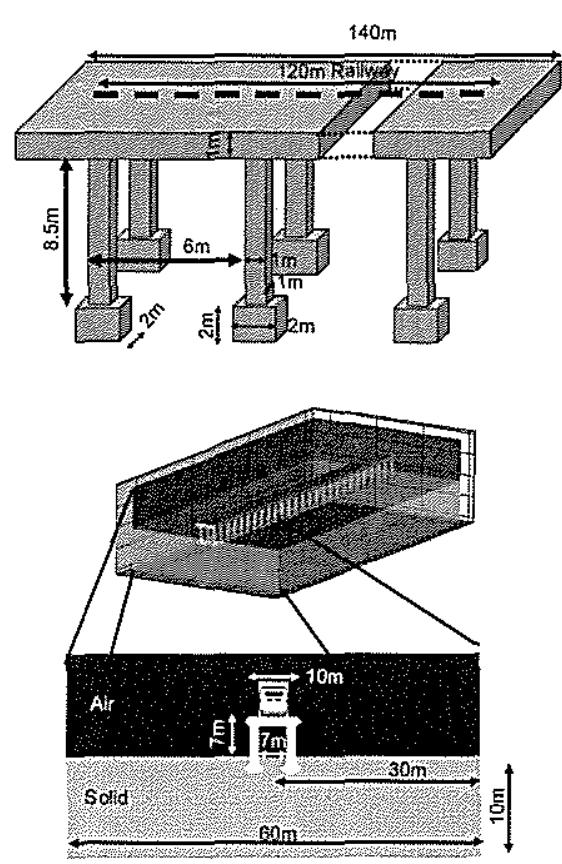

Figure 13 Schematics of elevated bridge model. 


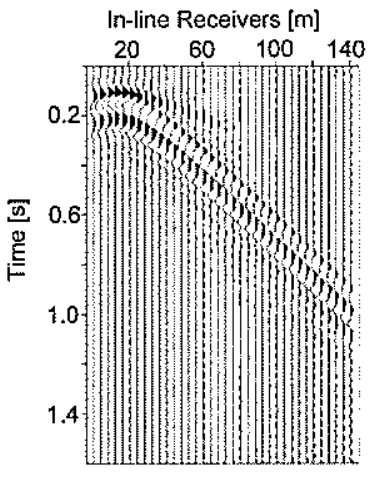

Figure 14: Waveforms of synchronized force and homogeneous media.

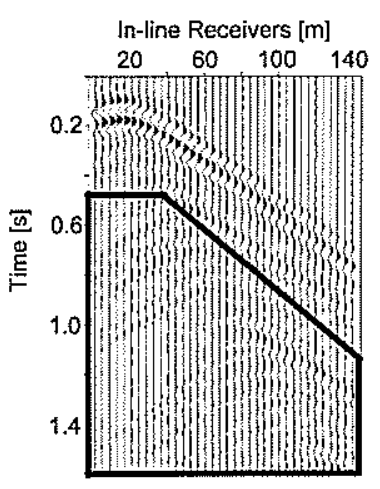

Figure 16: Waveforms of synchronized force and inhomogeneous media.
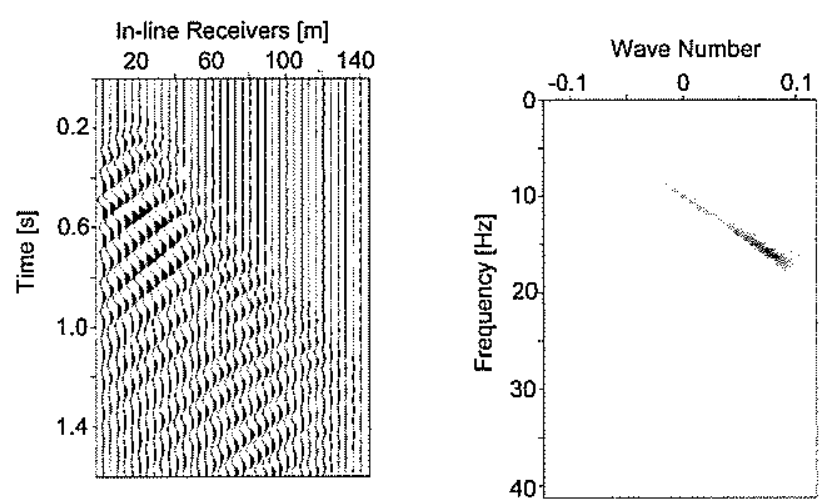

Figure 18: Waveforms of synchronized force and elevated bridge.

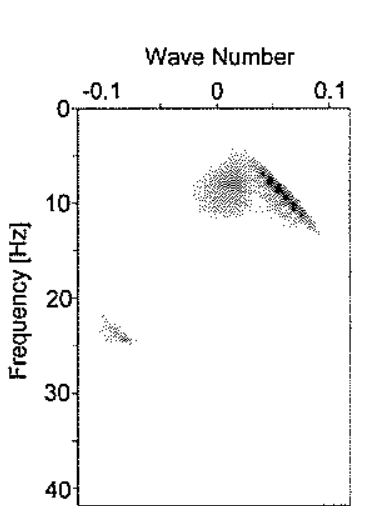
trum of Fig14.

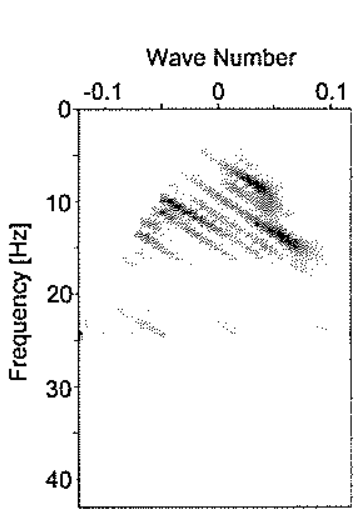
trum of Fig16. trum of Fig18.

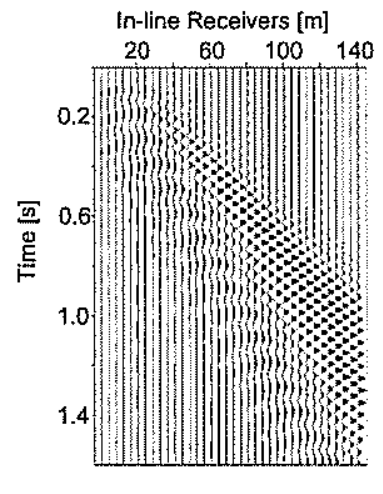

Figure 20: Waveforms Figure 15: FK spec- of not synchronized force

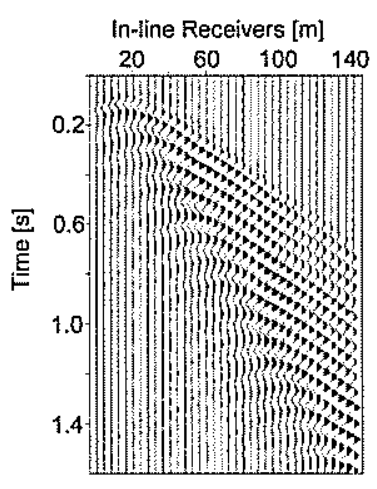

Figure 22: Waveforms Figure 17: FK spec- of not synchronized force

and inhomogeneous media.
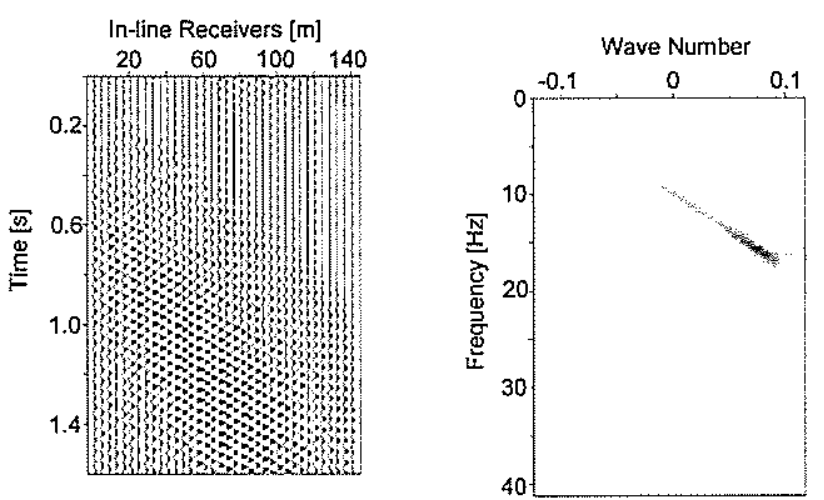

Figure 19: FK spec-

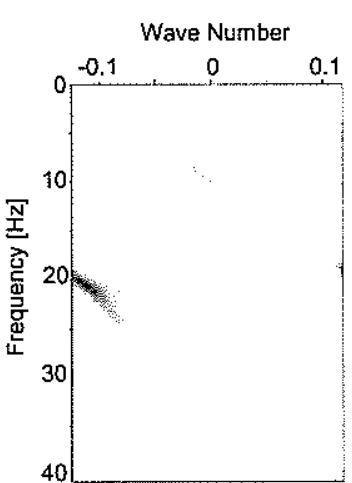

Figure 21: FK spectrum of Fig20.

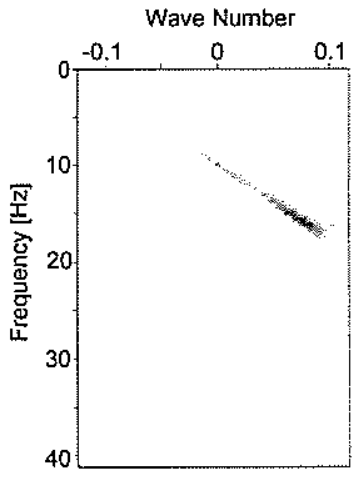

Figure 23: FK spectrum of Fig22.
Figure 24: Waveforms of not synchronized force and elevated bridge.
Figure 25: FK spectrum of Fig24. 


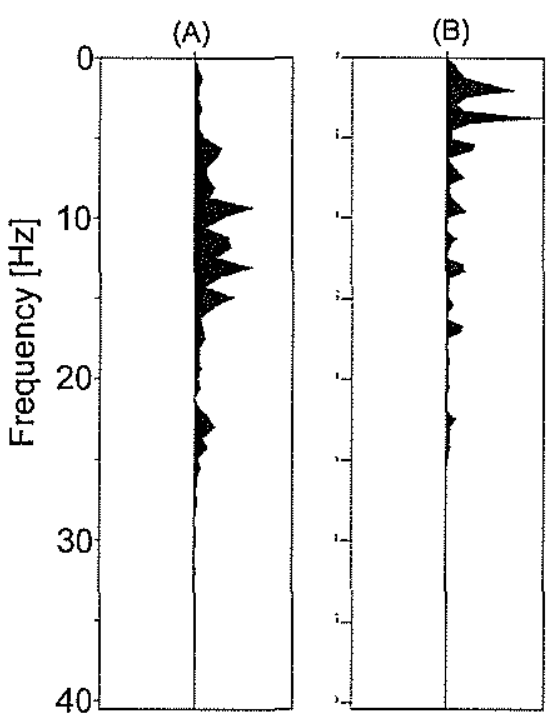

Figure 26: (A) : Spectrum at wave number 0 in Fig17, (B) : (Vs parameter under the railroad) / (Train speed).

\section{Conclusions}

We investigated the reason of the ground vibration generated by train. Comparing to qualitative properties of field data, we were able to classify the types of ground vibration by numerical simulations. In case2 to case6, They also show Doppler effect. Thus they are important classification of the ground vibration generated by train. We will have to quantitatively investigate problematic area. We summarize following terms.

- We analyzed the field data in bank area. They observe Doppler effect in the ground vibration. We realized that numerical models generate ground vibration showing Doppler effect.

- 3D numerical simulation shows 2 reasons of occurrence of ground vibration in bank area. They are inhomogeneous media and not synchronized force from train. You could improve media and force from train homogeneous, ground vibration did not occur. But it is impossible. We will have to quantitatively compare case 2 to case 4 in future.

- We also carried out numerical simulation in case of elevated bridge model. We realized that the ground vibration is generated since bridges touch ground discretely. Even if media or force from train could be improved homoge- neous, the ground vibration would occurre in elevated bridge area.

\section{Future work}

We need to develop the simulation tool evaluating wave filtering quantitatively. To make clear the mechanisms of ground vibration in all near railroad area, inmohogenesity of force from the train and boundary problem between train body and ground will have to be decided. In short, Force at ground surface to input waveform is not made clear. If we decide input waveform, we will improve our understanding by comparing to quantitative vibration level of the field data.

\section{Acknowledgments}

We wish to thank Hayashi Ko-ichi for permition us the viscoelastic wave equation finite difference code including parallelization. Thanks to Mitsuduka Takashi for measuring field vibration data.

\section{References}

1) Yoshioka, O, Ashiya, K (1995), A Dynamic Model on Excitation and Propagation of Shinkansen-Induced Ground Vibrations, Society of Exporalation Geophysics Japan, 48, 299315. (in Japanese)

2) Hayashi, K (1999), Variable Grid Finitedifference Modeling Including Surface Topograghy, Department of Erth, Atmospheric, and Planetary Sciences in the partial fulfillment of the requirements for the degree of Master of Sciences at the Massachusetts Institute of Technology.

3) Kamei, R, Matsuoka, T, Hato, M (2004), Geophysical Model for Gas Hydrate Layer using Anisotropic Random Medium, 110the Society of Exporalation Geophysics Japan proceeding, 75-78. (in Japanese)

4) Aki, K., Richards, P.G., Quantitative seismology: Theory and methods, vol. 2, San Francisco, W. H. Freeman and Company, 749. 